\title{
9 Traumatisierung: sexueller Missbrauch
}

\author{
Wolfgang Harth
}

Schwere Traumatisierungen sowie deren Langzeitfolgen werden durch sexuellen Missbrauch, körperliche oder emotionale Vernachlässigung (Deprivation) oder körperliche Misshandlung verursacht.

Sexuelle Traumatisierung bei Männern war bisher ein gesellschaftliches Tabuthema ist aktuell jedoch immer mehr in den öffentlichen Fokus gekommen.

\subsection{Definition}

Als sexueller Missbrauch werden sexuelle Handlungen definiert, welche die sexuelle Selbstbestimmung einer Person verletzen, die noch nicht ein bestimmtes Alter erreicht hat, in einer besonderen Beziehung zu dem Täter steht oder sich körperlich oder mental nicht widersetzen kann (Egle et al. 1997).

\subsection{Häufigkeit}

Im Bundesgebiet wurden 2008 insgesamt 56.784 Straftaten gegen die sexuelle Selbstbestimmung erfasst, wobei der absolute Anteil der Vergewaltigungen 7.292 sowie der sexuelle Missbrauch von Kindern 12.052 Fälle betrugen.

Eine nationale Telefonbefragung in den USA 2001-2003 zeigte, dass 1 von 15 Erwachsenen in den USA (2,1 Mio. Männer und 11,7 Mio. Frauen) sexuellen Missbrauch erfahren haben (Basile et al. 2007). Sozialwissenschaftliche Untersuchungen gehen aber von einer weitaus größeren Dunkelziffer aus. Die Prävalenzziffern (Spencer u. Dunklee 1986) von sexuellem Missbrauch ergeben eine große Streubreite mit Angaben von 9-38\% bei Frauen und von 9-16\% bei Männern. Ein Vergleich ist aufgrund von unterschiedlichen Beobachtungszeiträumen der einzelnen Studien sowie der nicht einheitlichen Definition von sexuellem Missbrauch nur bedingt möglich. 
Sicher ist jedoch, dass aufgrund der übereinstimmend hohen Prävalenz von sexuellem Missbrauch bewusst oder unbewusst jeder Mediziner mit diesem Problembereich konfrontiert ist und dabei vor besondere Anforderungen der venerologischen Erkrankungen sowie Diagnostik und Therapie von psychosomatischen Störungen gestellt wird.

\title{
9.3 Einteilung
}

Die medizinischen Fragestellungen nach einem sexuellen Missbrauch sind sehr unterschiedlich, und stellen immer eine besondere Situation und Herausforderung dar, in der strukturiert und ruhig vorgegangen werden muss. Die praktische Erfahrung hat gezeigt, dass außer akuten auch die verdeckten Langzeitfolgen sowie Folgen von entbehrlichen Untersuchungen bei nicht bestätigten Verdachtsdiagnosen beachtet werden müssen. Es lassen sich speziell drei Schwerpunktgruppen unterscheiden (s. Tab. 1).

\section{Tab. 1 Übersicht: Sexueller Missbrauch}

\author{
akute direkte Folgen von sexuellem Missbrauch \\ Verletzungen \\ sexuell übertragene Erkrankungen \\ Gravidität (bei Frauen) \\ psychische Symptomatik \\ Langzeit-Folgeerscheinungen von sexuellem Missbrauch \\ körperliche Funktionseinschränkungen \\ psychosomatisch-psychiatrische Erkrankungen \\ Imitationen und Fehldiagnosen \\ spezifische Dermatosen unter dem Bild eines sexuellen Missbrauchs \\ iatrogen induzierte, reaktiv psychische Symptomatik
}

\subsection{Klinische Befunde}

Als akute Folge finden sich direkt im Anschluss an einen Missbrauch Verletzungen im Genital- häufiger im Körperbereich.

Körperliche Leitsymptome sind Verletzungen an untypischen Stellen (Gesäß, Rücken, Genitale, Innenflächen der Oberschenkel) und auffällige Verletzungsmuster.

Nach entsprechender Inkubation kann ggf. das gesamte Spektrum von sexuell übertragenen Geschlechtserkrankungen (STD) nachgewiesen werden. In der Literatur liegen hierzu besonders Daten für die Gonorrhoe, Herpes genitalis, Condylomata accuminata, Chlamydien Urethritis, Pedikulosis pubis, Skabies und HIV vor. Hierbei sind die unterschiedlichen sexuellen und nicht-sexuellen Übertragungsmöglichkeiten zu beachten. 
Als weiteres Problemfeld können spezifische Dermatosen mit verwechselbarer Morphologie einen sexuellen Missbrauch vortäuschen. Die differenzialdiagnostische Abklärung muss in dieser für alle Beteiligten vorliegenden Ausnahmesituation besonders behutsam erfolgen, um iatrogen traumatisierende Prozeduren zu vermeiden.

Schon die Lokalisation einer Dermatose im Genitalbereich kann ebenso wie eine atypische Morphologie, falsch positive Labortests oder falsche Anamnese im Rahmen neurotisch-psychotischer Erkrankungen, zur fälschlich angenommenen Verdachtsdiagnose eines sexuellen Missbrauchs führen (s. Tab. 2). Imitationen erfordern eine sorgfältige aber auch vorsichtige Vorgehensweise im Vorfeld der Diagnosesicherung.

\section{Tab. 2 Imitationen und Fehldiagnosen: Differenzialdiagnosen sexueller Missbrauch}

\begin{tabular}{l} 
A Dermatosen im Genitalbereich \\
allergisches-toxisches Kontaktekzem (Phytodermatitis) \\
endogenes Vulvaekzem \\
Windeldermatitis \\
Hämorrhagien, Vaskulitis \\
blasenbildende Dermatosen \\
Lichen sclerosus atrophicans (hämorrhagisch nach Minimaltrauma mit Toilletenpapier) \\
Lichen planus \\
Psoriasis \\
\hline B Infektionen \\
bakteriell (Steptococcen) \\
Mykosen (Candidose) \\
Viren (Varizellen, Herpes, Condylomata acuminata) \\
Parasiten (Würmer) \\
\hline C Neoplasien \\
Karzinome \\
Sarkome \\
\hline D kongenitale Fehlbildungen \\
Gefäßmissbildung (Haemangiom) \\
Epispadie \\
Syndrome (Klippel Trenaunay) \\
\hline E Trauma \\
Irritationen \\
Sturz, Unfall (PKW) \\
kulturell (Beschneidung) \\
F Systemerkrankungen \\
Morbus Crohn \\
Megakolon \\
Fisteln \\
\hline
\end{tabular}




\subsection{Psychopathologische Symptome}

Akutfolgen von sexuellem Missbrauch sind sehr unterschiedlich und hängen vorwiegend vom Alter der Betroffenen, der Intensität und Bedrohlichkeit des Missbrauchs, Beziehung zum Täter und besonders bei Kindern auch von der Häufigkeit und Länge des Zeitraums der Traumatisierung ab. Direkt im Anschluss an einen sexuellen Missbrauch werden bei Kindern am häufigsten Änderungen der psychischen Verhaltensweisen wie Entwicklungsstörungen, Lernstörungen, exzessive Sexualisierung einschließlich Masturbation, Berührung der Genitalien von anderen Kindern, Weglaufen, Schule schwänzen und Enuresis, Selbstverletzung, Schlafstörungen, Essstörungen oder suizidale Tendenzen berichtet. Manche misshandelten Kinder zeigen charakteristische Auffälligkeiten in der Interaktion wie z.B. ein sog. eingefrorenes Lächeln oder eine eingefrorene Wachsamkeit. Auffällig ist häufig eine Störung der Nähe-Distanz-Regulation. Hinzu kommen Ängste in Situationen, die an den Misshandlungskontext erinnern wie beispielsweise Baden und Duschen oder Ängste bei körperlichen Untersuchungen.

Bei missbrauchten Erwachsenen sind als Akutfolge häufig eine Depression und Minderung des Selbstwertgefühls, akute Belastungsstörungen, Angst, Scham und Schuldgefühle zu beobachten.

Abzugrenzen hiervon sind die Langzeit-Folgeerscheinungen die im späteren Verlauf meist erst nach Jahren oder Jahrzehnten auftreten. Ein sexueller Missbrauch in den kindlichen Entwicklungsjahren hat Folgen für das ganze spätere Leben. Als Bewältigungs- bzw. Abwehrmechanismus der traumatisierenden Situation kommt es bei den Patienten zu posttraumatischen Belastungsstörungen, Spaltungsphänomenen und es können langfristig dissoziative Störungen bis zu Borderline-Persönlichkeitsstörungen oder Psychosen auftreten. Häufig besteht als Langzeitfolge nach sexuellem Missbrauch (Springer et al. 2007) auch nur eine unspezifische depressive Symptomatik $(83 \%)$ sowie weiterhin Schlafstörungen, Kopfschmerz, Pelipathie, chronische Gastritis, somatoforme Schmerzstörungen, Phobien, Essstörungen, Suchterkrankungen, Suizidgedanken, Selbstverletzungen, häufige Inanspruchnahme von ärztlicher Hilfe, erhöhte Scheidungsraten, sexuelle Störungen einschließlich Promiskuität oder Prostitution.

\section{Bei Artefaktpatienten, Selbstverletzungen im Bereich der Unterarme, Border- line-Störungen oder Anorexia nervosa sollte an psychosomatische Langzeit- folgen eines sexuellen Missbrauchs in der Vorgeschichte gedacht werden.}

Den dritten Problembereich stellen iatrogene induzierte psychische Störungen durch eine anfängliche jedoch letztendlich falsche Verdachtsdiagnose auf sexuellen Missbrauch dar.

Allgemein gewarnt werden muss vor zu schnell ausgesprochenen Verdachtsdiagnosen und der damit verbundenen Störung des Familiengefüges, die bis hin zur Ehescheidung führen kann. So können allein die Verdachtsdiagnose und entsprechende diagnostische Maßnahmen zwecks Ausschluss eines sexuellen Missbrauchs zu Misstrauen und Kränkungen aufseiten der Betroffenen, Partner oder der Familie führen und reaktiv psychische Störungen auslösen. 
Weiterhin sollten negative Äußerungen über Missbraucher des Kindes vermieden werden, da die Kinder insbesondere dann eine ambivalente Beziehung zu diesen haben, wenn sie die Bezugspersonen sind. Schuldvorwürfe, aggressive Konfrontationen oder Vorhaltungen an die Eltern sind kontraindiziert.

\subsection{Diagnostik}

Der Nachweis eines sexuellen Missbrauchs ist sehr schwierig und nur durch aufwendige Anamneseerhebung, körperliche Untersuchung, medizinische Tests und eingehende psychologische Untersuchung möglich. Dies wird normalerweise interdisziplinär in regionalen Spezialzentren meist in Kooperation der Ermittlungsbehörden und spezialisierter Kliniken oder Zentren durchgeführt. Gelegentlich wird auch ein Missbrauch vorgetäuscht, was ausgeschlossen werden muss.

Sexuell übertragene Infektionen und charakteristische Verletzungen im Genital- und Analbereich sind wichtige Leitsymptome zur Sicherung eines sexuellen Missbrauchs.

Die Befunderhebung bei Verdacht auf sexuellen Missbrauch ist immer dann rasch geboten, wenn die Möglichkeit besteht, kurz nach der Tat Spermaspuren zu asservieren oder akute Verletzungen behandelt werden müssen. Der Arzt wird weiterhin zum Ausschluss einer sexuell übertragenen Erkrankung hinzugezogen.

Bei Kindern finden sich generell sehr selten Geschlechtserkrankungen. Bei dem Vorliegen einer der klassischen Geschlechtserkrankungen im Kindesalter sowie spezifischem Befund ist dies mit hoher Wahrscheinlichkeit auf einen sexuellen Missbrauch zurückzuführen.

Besondere Schwierigkeiten bereiten in diesem Zusammenhang die Gruppe Sexually Transmitted Diseases (STD). So kann prinzipiell bei genitalen Papillomavirusinfektionen oder Herpes-Infektionen eine Übertragung durch Schmierinfektion stattfinden, jedoch muss auch die Frage nach einem sexuellen Missbrauch ausgeschlossen werden. Untersuchungen an größeren Patientenzahlen führten zu der Feststellung, dass bei der Mehrzahl von Kindern mit Condylomata acuminata ein sexueller Missbrauch nicht direkt nachweisbar ist und andere Ursachen möglich sind (Gross 1992).

Atypische Befunde oder protrahierte Behandlungsverläufe bei psychisch auffälligen Komorbiditäten können als Spätfolge anfänglich oftmals nicht direkt einem sexuellen Missbrauch zugeordnet werden, da dieser und dessen Folgen von den Patienten über einen längeren Zeitraum weder ausgesprochen noch thematisiert werden, oder ein unbewusster Zusammenhang vorliegt. Patienten mit einer Erfahrung von sexuellem Missbrauch zeigen oftmals große Widerstände, diese Information preiszugeben. Oftmals können die Patienten sich an den verdrängten Missbrauch nicht erinnern, und wird dieser erst im Rahmen einer Langzeitpsychotherapie bewusstseinsnah. Besonders bei einer Artefakterkrankung, posttraumatischen Belastungsstörungen, dissoziativen Störungen oder Borderline-Persönlichkeitsstörungen sollte eine weiterführende tiefenpsychologische Diagnostik unter Beachtung eines sexuellen Missbrauchs in früheren Entwicklungsjahren in Erwägung gezogen werden.

Ein spezifischer Test oder eine Verhaltensweise zum Ausschluss oder zur Bestätigung von sexuellem Missbrauch auf körperlicher oder psychischer Ebene existiert nicht. Wichtig sind Ruhe und Zeit in der akuten Krise, Vermeidung von Schuldvorwürfen 
oder aggressiven Konfrontationen mit den Eltern. Das Einschalten der Polizei ist eine folgenschwere Entscheidung, die nicht im Affekt und möglichst im Team getroffen werden sollte.

\subsection{Therapie}

Sexualmedizinische Fragestellungen besonders im Zusammenhang mit einem möglichen sexuellen Missbrauch bedürfen in allen Fällen einer psychosomatischen Grundversorgung oder intensiveren psychotherapeutischen Maßnahmen.

An erster Stelle steht der Schutz der Patienten. Ein interdisziplinäres Vorgehen zielt darauf ab, zunächst Sicherheit, Schutz und Vertrauen herzustellen. So muss einerseits bei Imitationen behutsam mit Zurückhaltung die notwendige Ausschlussdiagnostik erfolgen, jedoch andererseits bei akut stattgefundenem Missbrauch konsequent gründlich aufgedeckt und gehandelt werden; möglicherweise bei Kindern unter Einbezug des Jugendamtes. Sexuell übertragene Erkrankungen müssen entsprechend der Leitlinien therapiert werden.

Bei Langzeitfolgen muss ein Augenmerk auf verdeckte unbewusste Zusammenhänge gerichtet werden, und die Scheu zur Einleitung einer kombinierten psychosomatischen Vorgehensweise als Standardtherapie abgelegt werden.

Eine Überpsychologisierung ist dabei zu vermeiden. Nicht jede Belastung muss relevant sein oder ausführlich durchgearbeitet werden. Manche Patienten haben eine stabile Abwehrleistung als Schutz gegenüber der Traumatisierung aufgebaut. Ein forciertes Besprechen oder Erinnern an die Traumatisierung kann zu einer Überflutung mit negativen Affekten und psychischer Destabilisierung der Patienten führen. Liegt eine psychische Störung vor und zeigt sich im Verlauf ein sexueller Missbrauch in der Vorgeschichte, darf keine fokussierte Reduzierung oder monokausale Verursachungsannahme erfolgen und es müssen weitere psychische Aspekte oder Persönlichkeitsstörungen beachtet werden.

\section{Literatur}

Adams JA, Harper K, Knudson S, Revilla I (1996) Examination findings in legally confirmed child sexual abuse. Pediatrics 97, 148-150

Basile KC, Chen J, Black MC, Saltzman LE (2007) Prevalence and characteristics of sexual violence victimization among U.S. adults, 2001-2003. Violence Vict 22, 437-448

Bays I, Jenny C (1990) Genital and anal conditions confused with child sexual abuse trauma. Am I Dis Child 144, $1319-1322$

Bundesministerium des Innern (Hrsg.) (2009) Polizeiliche Kriminalstatistik 2008. (http://www.bmi.bund.de/cae/ servlet/contentblob/541740/publicationFile/26704/PKS2008.pdf, abgerufen am 14.09.2011)

Davies AG, Clay IC (1992) Prevalence of sexuallly transmitted disease infection in woman alleging rape. Sex Transm Dis 19, 298-300

Egle T, Hoffmann SV, Joraschky P (1997) Sexueller Mißbrauch, Misshandlung, Vernachlässigung. Stuttgart New York: Schattauer Verlag

Gross G (1992) Condylomata acuminata in der Kindheit-Hinweis für sexuellen Mißbrauch. Hautarzt 43, 120-125

Harth W, Linse R (2000) Dermatological symptoms and sexual abuse: a review and case reports. I Eur Acad Dermatol Venerol 14, 489-494

Kos L, Shwayder T (2006) Cutaneous manifestations of child abuse. Pediatr Dermatol 23, 311-320 
Ramos S, Lukefahr JL, Morrow RA, Stanberry LR, Rosenthal SL (2006) Prevalence of herpes simplex virus types 1 and 2 among children and adolescents attending a sexual abuse clinic. Pediatr Infect Dis | 25, 902-905

Senn TE, Carey MP, Vanable PA, Coury-Doniger P, Urban MA (2006) Childhood sexual abuse and sexual risk behavior among men and women attending a sexually transmitted disease clinic. I Consult Clin Psychol 74, 720-31

Sinal SH, Woods CR (2005) Human papillomavirus infections of the genital and respiratory tracts in young children. Semin Pediatr Infect Dis 16, 306-316

Sommers MS (2007) Defining patterns of genital injury from sexual assault: a review. Trauma Violence Abuse 8, $270-280$

Spencer MJ, Dunklee P (1986) Sexual abuse of boys. Pediatrics 78, 133-138

Springer KW, Sheridan I, Kuo D, Carnes M (2007) Long-term physical and mental health consequences of childhood physical abuse: results from a large population-based sample of men and women. Child Abuse Negl $31,517-530$

Young MS, Harford KL, Kinder B, Savell JK (2007) The relationship between childhood sexual abuse and adult mental health among undergraduates: victim gender doesn't matter. | Interpers Violence 22, 1315-1331 